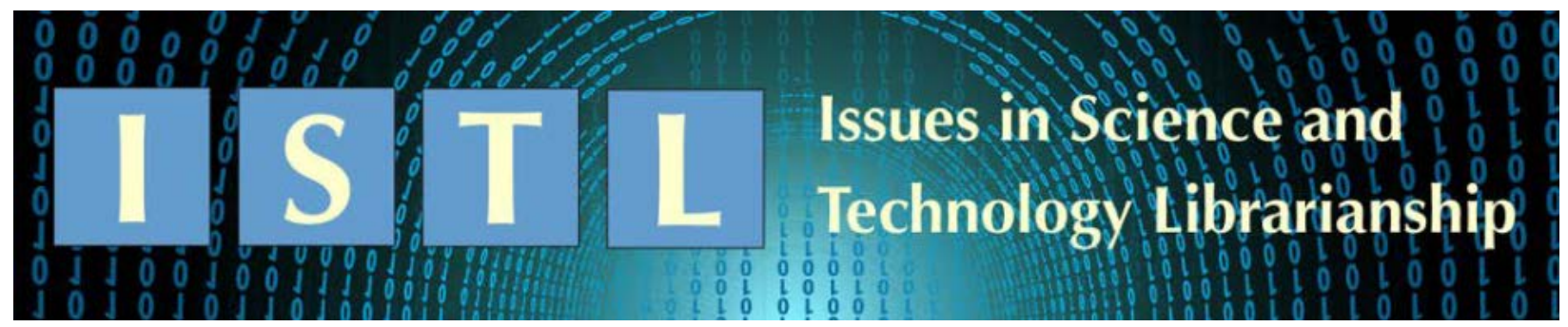

\title{
Science Librarianship and Social Justice: Part Two Intermediate Concepts
}

\author{
Jeffra D. Bussmann \\ California State University, East Bay \\ jeffra.bussmann@csueastbay.edu \\ Isabel M. Altamirano \\ Georgia Institute of Technology, Atlanta \\ isabel.altamirano@library.gatech.edu \\ Samuel Hansen \\ University of Michigan Libraries \\ hansensm@umich.edu \\ Nastasha E. Johnson \\ Purdue University Libraries and School of Information Studies \\ nejohnson@purdue.edu \\ Gr Keer \\ California State University, East Bay \\ gr.keer@csueastbay.edu
}

\section{Introduction}

This is the second column of four in a series that aims to open a dialog around equity, diversity, and inclusion (EDI) concepts, definitions, and examples in both the library and science contexts. [EDI Resources: ALA, ACRL, SLA, STS] This column builds upon the 16 foundational concepts discussed in the first column and provides scaffolding to discuss increasingly complex and nuanced terms in upcoming columns.

The concepts that appear in this second column begin to delve deeper into specific aspects of social justice and express social justice's range. Many of the concepts in this column have multiple possible definitions and, while we are not able to cover all of them, we aim to provide a representative sample. If you want to delve deeper into any of these concepts, there exist many additional resources, academic and otherwise, which you can access through your local academic or public library. A good place to start is within the Libraries Respond pages published and curated by ALA (http://www.ala.org/advocacy/diversity/libraries-respond). 
As a reminder, the goals of the column are

- To engage readers in meaningful and intentional conversations around EDI and ask them to reflect on their own practices

- To expose readers to scaffolded social justice concepts as pertinent to serving and teaching people of diverse backgrounds

- To demonstrate the relevance of social justice concepts by using examples that intersect with science librarianship

As a group of five librarians from different institutions, there is no single positionality statement that could cover all of us. That said, the work behind these columns is driven at least in part because of our identities and lived experiences including non-binary, Latina, Black, White, queer, and autistic librarians. We have come together as a group to bring conversations about justice to the forefront of science librarianship.

For most of the definitions and examples, we are building off of our own lived experiences, education, and observations. However, we realize that our perspectives are limited, and we acknowledge and welcome different voices. For example, with Model Minority, because none of us have Asian American or Asian heritage, we consulted with six Asian/Asian American colleagues about our draft definition and examples. Their names are listed in our acknowledgements. Based on their feedback, we modified it to more closely align with their lived experiences.

This work will likely push some readers outside of their comfort zone, but the work is intended to spur conversation and not to alienate. Thus, the authors encourage readers to use the discussion questions at the end for self-reflection and/or to help start a conversation in their library or organization.

For more information about why and how we are writing this column and our target audience, please refer to the introduction of our first column.

\section{Intermediate Concepts}

\section{Accessibility}

How well something is designed to allow for as many people as possible, with as many different embodied experiences as possible, to engage with or use it without needing to request modifications. The quality of access or the ability to reach a resource or tool; in education, accessibility can refer to the ability to access materials and resources that enable everyone's ability to learn, specifically targeted to combat ableism and assist those who are disabled. In the library and information science context, access and accessibility are sometimes conflated to refer to public access to publications and data. This is a different use of the term.

Library Example: The library embarks on an audit of their physical location and their website to make sure that it is accessible. They make sure their physical location not only adheres to the Americans with Disabilities Act requirements, but also takes into account community needs that 
have been provided via the Suggestions box and an anonymous survey. They fix any errors that are illuminated by accessibility web checkers to make sure their website follows the Web Content Accessibility Guidelines (WCAG), and they test the usability of their desktop and mobile web versions with commonly used screenreaders. They commit to building accessibility into all future building and website projects.

Science Example: In an environmental science class, which is a requirement for graduation, the professor is teaching remotely/online. The professor assigns team members. The first assignment involves going to a park and taking water and soil samples. The professor notices that the Team $B$ report has no input from a student, Guy. When the professor contacts the student, Guy asks her if the samples could be mailed to his home, where he can analyze them. He informs the professor he has limnophobia and hylophobia (tevens 2019). Realizing her class is not fully accessible, the professor contacts the Mental Health Department in order to ask for advice about how to alter the assignment in order to make sure Guy and future students have options for how and where they collect and analyze samples.

\section{Ageism}

Discrimination, attitudes, actions, or policies that limit the participation of persons based on their age rather than their skill or talent. There are some legal protections for those 40 years or older against hiring discrimination, but functionally ageism affects people of all ages (U.S. Department of Labor n.d.).

Library Example: The department head of Access Services needed to nominate one employee to be responsible for their department's use of a new ethics reporting tool. She decided on the new hire, who is young, but did not send them to the university training on the tool because she believes young people are naturally technology-savvy and do not need technology training. The new hire struggled to learn the tool on their own.

Science Example: An opportunity comes to the biology department for a new sponsored lab. An experienced researcher, potentially 5 years shy of retirement, submits a proposal for use of the lab. The proposal review board considers her too old to learn how to use the new equipment that will be installed in the lab space and immediately removes her application from the review pile.

\section{Antiracist}

People, organizations, or philosophies that actively reject and intentionally work to dismantle

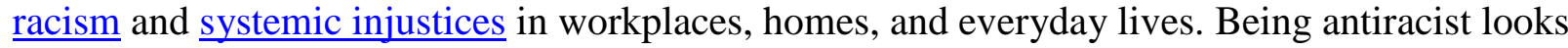
different depending on your identity and positionality (National Museum of African American History of Culture n.d.). It is important to acknowledge that to not be racist is not the same as being antiracist.

Library Example: The eugenics movement has a racist viewpoint and promotes the idea that European groups are superior and deserve to thrive and non-European groups are inferior and should be eliminated. The librarians reviewing books from the eugenics movement find the language abhorrent but also recognize the historical value of these works. They place an 
explanation in the catalog and on the print books to explain the background of the movement in order to highlight its racist and non-scientific nature, as well as including links to works the librarians added to the collection that refute the philosophy of the eugenics movement. The explanation and additions to the collection allow the books to stay in circulation while also providing important and necessary context.

Science Example 1: The tenure and promotion committee in the astronomy department was having heated conversation about the appropriateness of a large podcast audience as an impactful measure of scholarship for promoting a BIPOC faculty member. The chair of the committee stopped the conversation and reminded everyone that, "We don't agree on podcasts but we can agree on the importance of authentic storytelling and oral traditions in some communities and cultures.”

Science Example 2: Rushton and Templer (2012) published an article that claimed the results from psychological studies combined with animal studies evidence strongly indicated that people with darker skin color were prone to more aggression and sexual activity. The journal, Personality and Individual Differences, retracted the article after other scholars expressed their outrage, identified the article as racist, and provided evidence to negate the conclusions of Rushton and Templer (Sapunar 2020).

\section{BIPOC}

BIPOC stands for Black, Indigenous, and People of Color with an emphasis placed on the unique experiences of Black and Indigenous peoples in the structures and systems of North America and beyond; notably Indigenous is an umbrella term and does not specify tribe membership or legal category. BIPOC attempts to counter issues inherent to the approach of combining all non-white groups together, such as implying they all have the same experiences or suffer from the same injustices, while providing a way to discuss the larger non-white community without centering white people.

Library Example: When the library administration saw that hiring BIPOC library workers was less challenging than actually retaining and promoting them, the administrators developed a working group, with members from the campus HR and Title IX and campus institutional equity office, to address and respond to the unique challenges that BIPOC librarians and BIPOC student workers face in being seen, heard, and supported in library work and research.

Science Example: At a student chapter meeting of the National Society of Black Physicists, one of the members brought forward that their physics department's website, literature, and advertising materials used the phrase "People of Color" or the initialization "POC.” The NSBP student chapter then requested the department instead use the phrase "Black, Indigenous, and People of Color" and the initialization "BIPOC" in order to bring to the forefront the lived experience of their Black and Indigenous faculty members, students, and applicants, and to indicate they understood non-white people were not a monolith and did not deserve to be treated as one. 


\section{Cisgender}

A person who identifies with the sex and presumed gender assigned to them at birth.

Library Example: After taking the training provided by the campus LBTQ student alliance, a library faculty member began using her pronouns in her signature, previously not seeing the need for them because she is a woman who identifies as a woman. She now understands that, as a cis person, she also has a gender identity and her pronouns reflect it.

Science Example: In order to determine the impact of gender on the development of scientific identity, the Principal Investigator recruits both cisgender and transgender participants for a collaborative research project between the biology and psychology departments.

\section{Color Blind}

Often used to describe people who profess to "not see" skin color or ethnic origin. This reflects an ideology that proposes to treat everyone equally regardless of race or ethnicity and an assumption that all lived experiences are the same for everyone. Color blindness completely bypasses any existing marginalization or systemic injustices that exist by minimizing the lived experiences of people from non-dominant groups.

(Outside of a social justice context, color-blind can refer to a person who is unable to differentiate between certain colors, such as red and green, or can only perceive a limited range of coloration.)

Library Example: In an effort to regard all authors equally when choosing books for public displays, a library prohibited using the race of an author in decisions for which books to include, instead relying on measures they felt to be more race-neutral such as circulation numbers and inclusion in the Western canon. This is how there came to be no books by Blacks in their Black History Month display.

Science Example 1: A researcher at a science conference was presenting on the 2018 American Public Health Association policy statement declaring racialized police violence a public health issue (American Public Health Association 2018). A scientist in the audience objected to this perspective and stated that public health should not be racialized because "all lives matter."

Science Example 2: In the U.S., the participants involved in clinical trials related to heart failure are predominantly white men. These trials then heavily influence treatment regimes for all populations, not just white men. This has resulted in ACE Inhibitor regimes being one of the most commonly used treatments for all patients with hypertension and cardiovascular disease, despite the fact that a recent study has shown they to lead to poorer outcomes for Black patients (Ogedegbe et al. 2015). 


\section{Heteronormative and Cisnormative}

Interrelated worldviews that assume heterosexual and cisgender normative cultures, behaviors, and identities as the default. These worldviews automatically position all non-heterosexual and non-cis cultures, behaviors, and identities as abnormal or exceptional, effectively erasing and marginalizing them.

Library Example 1: A student comes to the reference desk for assistance finding information about the stability of American families over the last fifty years. The reference librarian directs the student to census records for marriage rates in the U.S. Neither the student nor the librarian considers the fact that same sex marriages have only been federally legally recognized since 2013 and are therefore not accurately reflected in census records. The student writes a paper based on this information, and receives an $\mathrm{A}$.

Library Example 2: During an analysis of the 500 romance novels on the Popular Reading shelves of the library where he worked, Henry realized only 1 involved a man-man pairing and even in that novel one of the characters in the couple was defined by traits traditionally held by society as feminine and the other character by traits traditionally held by society as masculine.

Science Example: When the blueprint of the new computer science building was released to the public, a petition was filed to protest the cisnormativity and heteronormativity of binary restrooms and baby-changing areas only in the women's restrooms. The blueprint excluded the interest of those who defied their presumptions of a gender binary.

\section{Imposter Syndrome}

A person's overwhelming sense of self-doubt and a fear that they will be discovered to be a fake or that they will be discredited, regardless of their accomplishments and expertise in their field.

Library Example: A librarian is encouraged to apply for a head of access services department by her colleagues. However, she feels that since she does not have extensive management experience, she is not qualified to lead the department. She declines to apply for the position, despite having the capacity to learn quickly on the job. The person who ultimately gets the position does not have any management experience.

Science Example: A student from a non-dominant group received a special invitation to present on their astrophysics summer research project but backed out at the last minute. The student later reported to their faculty member that they felt inadequate to make the presentation to the faculty and their fellow students.

\section{Microaggressions}

Frequently occurring indignities based on race, gender, cultural background, sexuality, or other marginalized identity. Their impact is felt in the cumulative effect of constant slights, misunderstandings, and messages that the targeted person is different, less respected, and less important. While some microaggressions are due to obliviousness rather than malice, they are 
often subtle or covert enough to fit within the spectrum of social norms and can be passed off as well-meaning mistakes in the case of intentional ill-will by the aggressor.

Microaggressions are often broken into three subcategories (Sue et al. 2007):

Microinsults are behaviors and language which convey a belief in denigrating stereotypes and demean the identity of non-dominant groups.

Microinvalidations invalidate, negate, and diminish the lived experience, thoughts, feelings, and reality of non-dominant groups.

Microassaults are explicit actions, biased language, and discriminatory behavior against non-dominant groups. These are not micro in content, but micro in situation or time; they usually happen in more private situations which allow the perpetrator some amount of anonymity to say or do what they would not do in public.

Library Example: In a library EDI committee, one member suggested that disabled people need to be included in the EDI charter, since there are no disabled members on the committee. An able-bodied library management employee, snorted at the suggestion and stated that disabled patrons have other advocates on campus and don't need to be included in any discussions for accessibility in the library.

Science Example: An African American first year PhD student in mathematics approached her advisor and told him that she was feeling excluded from activities in the otherwise all white department. Instead of recognizing that her lived experience could be different than his, the white professor responded with, “Don't worry, it isn't a race thing you see. It is just that you are new and the department is a very meritocratic group. Once you show yourself worthy, people will let you in."

\section{Model Minority}

A myth and a stereotype that a minoritized group is highly successful in some way, professionally, academically, socioeconomically, etc., particularly when compared to other minoritized groups. In the U.S., there is a Model Minority myth often associated with those of Asian heritage that suggests they are exhibiting the prototypic behavior that the dominant culture expects from minoritized people, e.g., docile, hard-working, affluent, non-confrontational, extremely smart, eager to please with no aspirations or capacity for leadership. This myth/stereotype can lead to an unreasonable expectation of specific kinds of success, e.g., academic, for members of the group, which can lead to a number of negative impacts. Furthermore, it flattens the range of different lived experiences of sub-groups and individuals within the group considered the Model Minority into a single narrative. In the U.S., this means the experiences of people of South Asian and East Asian heritage are considered interchangeable. It also means the experiences of people of Southeast Asian heritage are often erased as they face more significant systemic barriers and therefore do not fit the narrative of success. The idea of the Model Minority can be wielded as a racial wedge by the dominant culture holding up the Model Minority with their perceived success and expected behaviors and questioning why other minoritized groups have not achieved and do not act the same (Chow 
2017). Finally, this concept can also be used as an excuse to not address racism and oppression against groups seen as Model Minorities and to exclude them from anti-discrimination programs because they are seen as having already succeeded (Linshi 2014).

Library Example: When the library administration convened a career development workgroup, the Asian and Asian American library workers were excluded because the administration perceived them as sufficiently successful and not in need of additional professional development or networking opportunities. Furthermore, when the Asian and Asian American library workers decided to look for their own career development opportunities, they could not find many national conferences that included specific tracks for Asian and Asian Americans seeking leadership development opportunities, as is sometimes seen for other BIPOC librarians.

Science Example: The graduate students in the nanotechnology lab participated in a week-long retreat. The purpose was to get to know each other better and to have a chance to reflect on career plans after completing their graduate degrees. One of the visualization exercises was to draw yourself in ten years. Peter, a second-generation Chinese American, drew himself as CEO of a wearable nanotech company and explained his goals. The other participants snickered; the team leader asked for an explanation. The team members who had no Asian heritage only considered Peter a great tech geek and didn't see him as a leader. Peter was furious to be judged by racial assumptions and excluded from having aspirations.

Science Example 2: In a study of Asian American students at a high school in California, it was found that the students were well aware of the academic model minority stereotype about Asian American students being intrinsically talented in math and science. While people may judge this as a positive stereotype, many of the students "expressed anger at being judged by racial assumptions." Not only that, almost all of the students had experienced difficulties that were made invisible by the stereotype that Asian American students "are the model students, they do not experience failure, and their success comes easy to them.” (Wing 2007)

\section{Neutrality}

The stance that it is not only possible, but incumbent on individuals, organizations, or disciplines to remain impartial or unbiased in activities, thoughts, behaviors, situations, disagreements, etc., with respect to the events in the world or the values, power structures, and systems they are embedded within. Neutrality provides a way to stay silent and observe injustices instead of commenting or acting and making that silence seem to be a moral triumph instead of a moral failing.

Library Example: It would seem that cataloging, just saying what a book is about, could easily be classified as a neutral activity. Even if the cataloger themself was able to push aside their viewpoints, opinions, and lived experience, this neutrality would still be unattainable because the systems we use for cataloging are not neutral. The Dewey Decimal System rather famously provides Christianity 230-289 (220-229 are for the Bible) and Other Religions with only 290299, and while Dewey's failings are well known, the Library of Congress subject headings also show bias. For example, LCSH includes the assumption that the default person from the United States who has trained to go to space is both white and a man (Ros 2019). This can be seen by 
the set of subject headings that cover U.S. astronauts having a single general category for "Astronauts--United States," as well as specific categories for "African American astronauts” "Hispanic American astronauts," and "Women astronauts--United States" and most specifically a category for “African American women astronauts," but nothing for either men or white astronauts from the United States (Library of Congress 2020).

Science Example: A BIPOC chemistry student comes to their faculty advisor to ask for support in a research study examining the impact of chemical runoff in waterways that serve low-income urban areas, based on their experience growing up in Flint, MI. However, the professor refuses to give permission for the study and admonishes that they must maintain neutrality in order to do good science.

\section{Positionality v. Objectivity}

Positionality is the idea that every person's race, gender, ability, class, and other social alignments deeply influences their ideas, values, and understanding of the world around them. Objectivity, on the other hand, argues that a person can, and must, remain uninfluenced by their personal thoughts and beliefs while creating knowledge.

Positionality stands in opposition to objectivity's idea that the influence of human subjectivity can be excised from knowledge creation. In the academic context, this recognizes that every part of the research process, from what questions are posed to how participants are chosen and how data is analyzed, is affected by the position of the researcher. This is as true in the sciences as any other research areas.

Library Example: Recognizing that, because everyone has personal values and differing lived experiences, no one can be objective while writing a research guide, a librarian included a statement of their positionality with regard to the topics covered within the guide (Hansen 2020).

Science Example 1: In the midst of Black Lives Matter uprisings in protest of state sanctioned violence against Black people, science organizations and publishers that originally saw themselves and their members as objective and apolitical realized in light of widespread racism that objectivity is a myth. In response, they publicly acknowledged the positionality of journal editors, scientists, and researchers and participated in \#ShutdownSTEM (Parikh 2020; $\underline{\text { arXiv.org }}$ $\underline{2020}$ ).

Science Example 2: When applying for a grant on infectious diseases, the grant writer and principal investigator wrote in the proposal that study populations would include Black, Indigenous, and Asian subjects living in three zip codes. She included a positionality statement that acknowledged the influences of the authors' lived experiences in choosing the populations to study. One of the reviewers questioned the lack of objectivity implied by including a positionality statement. 


\section{Personal Pronouns}

Third person pronouns used to refer to a person present in conversation or in their absence that are chosen by the individual and may or may not align with visible identity or birth records. The pronouns she/her/hers and he/him/his are commonly used by people who have a binary gender identity (usually “woman” or "man”). Nonbinary people may use binary-aligned pronouns, or they may use nonbinary pronouns such as they/them/theirs, zie/zim/zir, ey/em/eir, or other pronouns. Incorrect use of personal pronouns can become a point of contention between cis (non-transgender) and transgender and nonbinary people when used incorrectly.

Library Example: The Dean sent out a "Welcome to our Library" email to introduce Morgan, the new Scholarly Research Librarian. The Dean incorporated Morgan’s personal pronouns (they, them, theirs) while describing their qualifications and previous work experience.

Science Example: Before recruiting participants for a research study on hypertension, the research group created an intake survey with a drop-down question about pronouns that only offered "he" and "she" as choices. One of the members of the research group challenged this by stating gender was not a variable in their study and they were only asking for pronouns in order to address participants by their pronouns. In order to make the question more inclusive of nonbinary genders, they changed their intake survey to create an open-ended question type for participants to share their pronouns.

\section{Unconscious Bias and Implicit Bias}

(These two phrases are used interchangeably to represent the same concept.)

A set of internalized and unexamined expectations held by an individual about people of different positionalities, reflected in associations and attitudes that lie below the level of conscious thought. They impact actions and attitudes without a person's knowledge or control. These biases, positive and negative, start to build during childhood through lived experiences and exposure to messages from family, friends, news, media, and society. There are many different types of unconscious/implicit bias (einers 2019).

Library Example: The most qualified candidate for the department head position was Paul, a little person. Despite all of his pertinent experience in Public Services, the hiring committee commented on not hiring him based on the idea that no one would listen to him because of his stature. Linda reminded everyone of their unconscious bias training and stated that height bias was affecting their viewpoints. Once Linda pointed out this behavior, the committee agreed to set aside the concerns about Paul's height affecting his leadership qualifications.

Science Example: A 2012 study showed that science faculty were less likely to hire a student as a laboratory research manager if the applicant had a name stereotypically thought of as a woman's than if the applicant's name was stereotypically thought of as a man's (Moss-Racusin et al. 2012). This was illuminated by using applications that were identical and randomly assigned the names John or Jennifer. This study relies on the idea of binary gender and the use of traditional Anglo names. 


\section{Underrepresented and Underserved}

Underrepresented refers to low numerical/statistical representation within a particular location and population, whereas underserved refers to a lower resource allocation compared to the sum of resources available.

Underrepresented Minorities (URM) is often used as an institutional label in higher education to describe students from marginalized communities, especially in the sciences.

In the US, there are higher education institutions that aim to serve particular populations. The US Department of Education defines seven specifically (U.S. Department of Education n.d.). Additionally, it is worth noting that words using 'under-' to describe particular student groups, such as URM, are being criticized and critiqued as being white-centered, deficit thinking, and removing distinct attributes of students (Williams 2020).

Library Example: The Collection Development team worked with Student Affairs to add books to the library. A recommendation was made to add works dealing with first-generation students, who are traditionally underserved; these students have many pressures to succeed and may not know how to navigate the structure of the academic world. One of the librarians objected because many graduates' children attend the university. Another member of the team countered that $20 \%$ of the student population are first generation students, the library only spends $5 \%$ of its available resources on materials to support this student group. Even though members of the team who were first-generation students said that if it were not for the support of their college library and/or library resources they would not have succeeded in college, the team was split on this issue.

Science Example: Engineering departments have long made efforts to boost the representation of women and racial minorities in the field. Some departments have seen this as a numbers game: just trying to drive their numbers of minoritized students up without asking themselves "how do we support these students?” Meeting the needs of the underrepresented students has led to creation of special outreach and retention efforts, while departments who try to meet the needs of underserved students have developed mentorship programs, identity-based learning communities, and need-based and presence-based scholarships and fellowships.

\section{Reflection Questions}

To engage further with these concepts and/or colleagues, we provide a few discussion questions:

1. Are any of our definitions different from your understanding of the concepts, and if so, how?

2. Given your lived experience, which of these concepts do you feel is most significant to your life?

3. Considering the departments that you liaise to, which of the concepts are most relevant in your work?

4. How can you apply these concepts to your work right now as a science librarian? 


\section{Acknowledgements}

The authors would like to gratefully acknowledge Tarida Anantachai, Andrew Carlos, Moon Kim, Lisa Ngo, Diana Wakimoto, and Lana Mariko Wood as consultants on the Model Minority section.

\section{References}

American Public Health Association. 2018. Addressing Law Enforcement Violence as a Public Health Issue: Policy No 201811. [accessed 2020 Aug 10]. https://www.apha.org/policies-andadvocacy/public-health-policy-statements/policy-database/2019/01/29/law-enforcementviolence.

arXiv.org. 2020. arXiv staff to participate in the \#strike4blacklives. arXiv.org Blog. [accessed 2020 Jul 6]. https://blogs.cornell.edu/arxiv/2020/06/08/strike4blacklives/.

Chow C. 2017. "Model Minority” Myth Again Used As A Racial Wedge Between Asians And Blacks. NPR KQED. [accessed 2020 Aug 7].

https://www.npr.org/sections/codeswitch/2017/04/19/524571669/model-minority-myth-againused-as-a-racial-wedge-between-asians-and-blacks.

Hansen S. 2020. Resources for Anti-Racism and Social Justice in the Mathematical Sciences. Aperiodical Irregululars. [accessed 2020 Aug 7]. https://aperiodical.com/2020/06/resources-foranti-racism-and-social-justice-in-the-mathematical-sciences/.

Library of Congress. 2020. LC Linked Data Service: Authorities and Vocabularies: Astronauts. Internet Archive - Wayback Machine. [accessed 2020 Aug 7].

https://web.archive.org/web/20200611154822/http:/id.loc.gov/search/?q=astronauts\&q=cs:http:// id.loc.gov/authorities/subjects\&start=1.

Linshi J. 2014. The Real Problem When It Comes to Diversity and Asian-Americans. Time. [accessed $2020 \mathrm{Jul}$ 6]. https://time.com/3475962/asian-american-diversity/.

Moss-Racusin C.A., Dovidio J.F., Brescoll V.L., Graham M.J. \& Handelsman J. 2012. Science faculty's subtle gender biases favor male students. Proceedings of the National Academy of Sciences. 109(41):16474-16479. DOI: 10.1073/pnas.1211286109.

National Museum of African American History of Culture. [n.d.] Being Antiracist. [accessed 2020 Aug 7]. https://nmaahc.si.edu/learn/talking-about-race/topics/being-antiracist.

Ogedegbe G., Shah N.R., Phillips C., Goldfeld K., Roy J., Guo Y., Gyamfi J., Torgersen C., Capponi L. \& Bangalore S. 2015. Comparative Effectiveness of Angiotensin-Converting Enzyme Inhibitor-Based Treatment on Cardiovascular Outcomes in Hypertensive Blacks Versus Whites. Journal of the American College of Cardiology. 66(11):1224-1233. DOI: $\underline{10.1016 / j . j a c c .2015 .07 .021 .}$ 
Parikh S. 2020. AAAS CEO Statement on \#ShutDownSTEM and Black Lives Matter. AAAS News. [accessed $2020 \mathrm{Jul}$ 6]. https://www.aaas.org/news/aaas-ceo-statement-shutdownstem-andblack-lives-matter.

Reiners B. 2019. 12 Unconscious Bias Examples and How to Avoid Them in the Workplace. Built In. [accessed 2020 Aug 7]. https://builtin.com/diversity-inclusion/unconscious-biasexamples.

Ros A. 2019. The bias hiding in your library. The Conversation. [accessed 2020 Jul 6]. http://theconversation.com/the-bias-hiding-in-your-library-111951.

Rushton J.P. \& Templer D.I. 2012. RETRACTED: Do pigmentation and the melanocortin system modulate aggression and sexuality in humans as they do in other animals? Personality and Individual Differences 53(1):4-8. DOI: 10.1016/j.paid.2012.02.015.

Sapunar A.L. 2020. Elsevier journal to retract 2012 paper widely derided as racist. Retraction Watch. [accessed 2020 Aug 10]. https://retractionwatch.com/2020/06/17/elsevier-journal-toretract-2012-paper-widely-derided-as-racist/.

Stevens S. 2019. 10 Traumatic Phobias Inspired by Nature. Treehugger. [accessed 2020 Aug 7]. https://www.treehugger.com/traumatic-phobias-inspired-by-nature-4869152.

Sue D.W., Capodilupo C.M., Nadal K.L. \& Torino G.C. 2008. Racial microaggressions and the power to define reality. American Psychologist. 63(4):277-279. DOI: 10.1037/0003066X.63.4.277.

U.S. Department of Education. [n.d.] ED: Lists of Postsecondary Minority Institutions. [accessed 2020 Aug 7]. https://www2.ed.gov/about/offices/list/ocr/edlite-minorityinst.html.

U.S. Department of Labor. [n.d.] Age Discrimination. [accessed 2020 Aug 7]. https://www.dol.gov/general/topic/discrimination/agedisc.

Williams T.L. 2020. “Underrepresented Minority” Considered Harmful, Racist Language. Blog@CACM. [accessed 2020 Aug 7]. https://cacm.acm.org/blogs/blog-cacm/245710underrepresented-minority-considered-harmful-racist-language/fulltext.

Wing J.Y. 2007. Beyond Black and White: The Model Minority Myth and the Invisibility of Asian American Students. Urban Review 39(4):455-487. DOI: 10.1007/s11256-007-0058-6. 